\title{
ORGANIC AMNESIA: A DIVERSITY IN DEFICITS
}

Christopher R. Madan

Department of Psychology, University of Alberta

\section{Abstract}

Organic amnesia is the loss of memory due to biological factors such as brain disorders, tumors, strokes, degenerative diseases, or any other of a multitude of other disruptions of neurological function. Memories are stored throughout the brain, and as a result damage to any localized brain region only causes limited memory deficits. Even in itself, organic amnesia can present in a variety of impairments across numerous memory systems within the brain. Several kinds of amnesia will be reviewed, including details of the corresponding deficits and suggestions of the likely affected memory system.

\section{Introduction}

Many people believe that memory disorders affect memory as a whole. However, this is not the case as memory is not a unitary faculty of the mind (Tulving and Schacter, 1990). Memory disorders do not simply affect all of memory, rather they only impair select memory systems. Amnesia is commonly caused by memory loss due to specific circumstances, mainly brain injury, illness, or psychological trauma (Passer and Smith, 2007, p. 257). As described by Budson and Price (2005), "memory loss is often the most disabling feature of many disorders, impairing the normal daily activities of the patients and profoundly affecting the families." Classically, amnesia is when "a person has problems recalling information from the past" (DANA Foundation, 2007), however, there are many other possible memory impairments.

Amnesic syndromes also have found their place in Hollywood, albeit usually becoming distorted. In movies such as 50 First Dates (2004) and Groundhog Day (1990), the amnesic syndromes presented are impossible to replicate in the real world, and are often cacophony of real symptoms viewed from an overly simplistic standpoint. This is not surprising as popular culture tends to take a "mix and match" approach when it comes to portraying amnesic syndromes (Baxendale, 2004). Amnesia has also been demonstrated on big screen recently through many other movies ranging from The Bourne Identity (2002) trilogy to The Eternal Sunshine of the Spotless Mind (2004) and Memento (2000). The representation of amnesia, along with other neuropsychological disorders, in popular culture is quite important as it can have a significant effect on public perceptions of those who actually exhibit the disorder.

Amnesia can be divided into many different categories, two of which are based on the proximal cause of the impairment: organic amnesia and psychogenic amnesia. The biological instances of the amnesia, organic amnesia, is usually caused by brain disorders, tumours, strokes, degenerative diseases, chronic usages of select drugs, temporal lobe surgery, or electroconvulsive therapy (DANA Foundation, 2007). The psychological memory disorders, psychogenic amnesia, are much rarer occurrences than organic amnesia and are characterized by a loss or dissociation of one's identity and past experiences (Simons and Graham, 2000). As suggested by Fujiwara, Brand, Kracht, Kessler, Diebel, Netz, and Markowitsch (2008), some instances of retrograde psychogenic amnesia may actually be organic amnesia where the "functional/ metabolic disturbances in memory-relevant brain structures may have been below thresholds for detection". In some cases, the opposite may also be true, where the organic amnesia is entirely or in part caused by a psychological mechanism (Fujiwara et al., 2008).

In the literature, the best known case of organic amnesia is patient H.M., who, in the 1950s, had an operation to relieve symptoms of severe epilepsy (Scoville and Milner, 1957; Corkin, 2002). The surgery involved his hippocampal complex and parts of his temporal lobes in both hemispheres. After surgery, H.M. developed profound amnesic symptoms even though his epilepsy had improved (Simons and Graham, 2000). Since Milner and colleagues' initial research with H.M., many more cases of hippocampal amnesia have been researched (Spiers, Maguire, and Burgess, 2001). Although bilateral hippocampal lesions are clearly causing profound amnesia, there is still controversy regarding the extent that unilateral hippocampal lesions cause amnesia (Spiers et al., 2001). H.M. is now typically used as a benchmark case in the study of the role of the medial temporal lobe in learning and memory (O'Kane, Kensinger, and Corkin, 2001).

Some diseases, such as Alzheimer's, Parkinson's, and Huntington's disease, which are not commonly associated with amnesia, also encompass memory impairments. Due to their neurodegenerative nature their resulting lesions are progressive, rather than focal. For example, in medial temporal lobe damage: H.M. was a focal lesion patient. On the other hand, in Alzheimer's disease, a neurodegenerative disease, patients tend to experience medial temporal lobe damage first, while later other structures become involved as well (Budson and Price, 2005). Focal lesions are caused by specific incident, such as surgery or trauma. In contrast, progressive lesions are caused by neurodegenerative diseases that progress, affecting more of the brain and nervous system throughout their course.

Amnesia can vary greatly between individuals. This can be explained by attributing different memory systems to the various anatomical brain regions that have come to be associated with the amnesic syndrome. The many disorders that give rise to amnesia, "including herpes encephalitis, severe hypoxia, certain vascular lesions, head injury, deep midline tumours, basal forebrain lesions, and occasionally early dementia" (Kopelman, 2002), also are associated with differences in the resulting symptoms. Depending on the type of amnesia and the suspected cause, treatments vary greatly. For example, "one drug, donepezil, has been found to improve thinking and memory in patients with Alzheimer's disease" (DANA Foundation, 2007). In spite 


\section{R. Madan / Eureka 2 (2011)}

of this, impairments to the episodic memory system are much more prevalent than any other memory system impairments (Spiers et al., 2001). "Episodic memory is our personal, conscious, explicit recollection of experienced events" (Budson and Price, 2001).

Organic memory impairments can be subdivided further according to many different classifications, including time (as seen in anterograde and retrograde amnesias), processes (encoding, consolidation, storage, retrieval), and content (depending on the affected memory systems). Memory systems can range from unconscious, as in priming, to what is commonly thought of as memory, the episodic memory system. The aetiologies of memory impairing disorders can vary significantly and are quite diverse in their features and mechanisms of their resulting deficits.

\section{Forms of Amnesia}

As mentioned, amnesia can also be differentiated by its effect relative to the onset of its appearance. If the memory deficit presents as a severe inability to retain new information, it is classified as anterograde amnesia (Kapur, 1999). On the other hand, if the impairment presents as a loss of memory that had already been acquired prior to the onset of the cerebral pathology, it is considered retrograde amnesia.

Anterograde and retrograde amnesia often occur concurrently. The most common pattern is characterised by a temporal gradient followed by Ribot's Law (Shimamura, 1992). Ribot's Law illustrates that there is a particular pattern where the "ability to learn new information is most impaired (anterograde amnesia), recently learned information cannot be retrieved (retrograde amnesia), and remote learned information is spared" (Budson and Price, 2001). Budson and Price (2001) also mention that "the period of retrograde amnesia can last anything from several hours (in the case of transient global amnesia) to several years (in the case of surgical excision of the temporal lobes)." This is not to say that amnesia is always graded as advocated by Squire, Clark, and Knowlton (2001), ungraded retrograde amnesia can occur from damage to structures of the lateral and anterior temporal lobe, while graded amnesia is often associated with medial temporal lobe damage.

Anterograde amnesia is primarily caused by damage to the hippocampal complex, impairing the individual's ability to acquire new memories (Simons and Graham, 2000). As described by Simons and Graham (2000), retrograde amnesia is much less localized physiologically, though the temporal lobe appears to be a principal storage site for human memory. Most research into amnesia has been focused on anterograde amnesia as it is considered to be more disabling in everyday life, leaving the study of retrograde amnesia widely neglected by researchers and clinicians until recently (Kapur, 1999). Several reasons for this neglect, include that clinicians and caregivers alike are often less aware of retrograde memory symptoms and that there are significantly fewer retrograde memory tests available to clinical neuropsychologists, as opposed to anterograde memory tests.
Kopelman (2002) suggests that anterograde amnesia can result from possible disturbances in all memory processes: faulty encoding, faulty consolidation, accelerated forgetting, and faulty retrieval. Various studies point to each of the above causes, likely due to differing pathological conditions causing the resulting amnesic syndromes. Faulty encoding is when there is a deficit in the process responsible for the initial representation of the information, such as in Korsakoff patients. Faulty consolidation occurs when the impairment prevents the information from being effectively transferred from the initial representation to a relatively permanent store, a prominent occurrence head injury patients. However, when the information is learned adequately learned, problems may still cause the deficits in retention, as in individuals with Alzheimer's disease. Lastly, faulty retrieval may also occur, where the impairment exists independently of the information acquisition.

Most instances of memory impairment are associated with a temporally limited retrograde amnesia (Banich, 2004, p. 328). The DANA Foundation (2007) reports that these temporary amnesia cases are due to the disruption of normal brain function for brief periods of time, without causing permanent brain damage as seen in closed head injuries. The most common cause of this disruption is due to a blow to the head, from a sports injury or automobile accident, interfering with the consolidation process (DANA Foundation, 2007). Other longer, localized, disruptions can be observed in transient ischemic episodes where there is a loss of language skills (anomia) while still retaining recollection of the word-finding task (Kapur, 1999).

The memory consolidation period is believed to be approximately 20 to 30 minutes, "the time it takes your brain to file an input or experience away in long-term memory" (McConnell, 1989, p. 279). When the consolidation process is not functional, new memories fail to be stored into longterm memory, resulting in anterograde amnesia. This was observed in H.M. who "could not recall what he had for lunch and could not even recall if he had lunch at all" (Shimamura, 1992), and referred to his condition as always "waking from a dream". Pharmacological factors can also have profound effects on the memory consolidation process (Gold, 2006). Gold (2006) explicates that these factors can be demonstrated principally through manipulations of hormonal levels as well as through protein synthesis inhibitors.

As explained by Banich (2004, p. 336), amnesics have very specific patterns of limited memory loss, and despite amnesics losing their ability to remember information, under certain special circumstances, their behaviours are indicative of learning and memory. Amnesics can still learn, though not always in the traditional sense. One way of assessing residual memory in the presence of amnesia, is to utilize a content-based classification of memory or memory systems (Ashby and O'Brien, 2005). Traditionally, amnesia is associated with an inability to recall previous events, a property of the episodic memory system, but there are also several other memory systems that can be affected by neurodegenerative diseases. With such classification, one can observe and describe memory loss in some memory domain and preserved memory in others. 


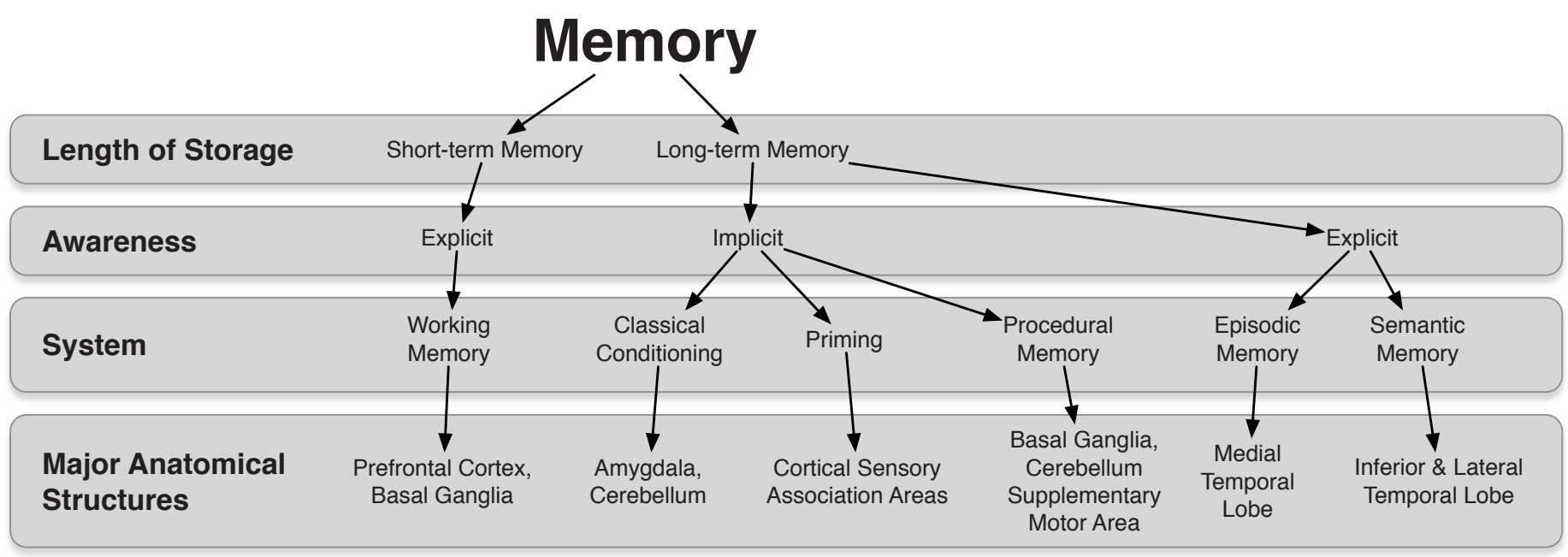

Figure 1. Memory System Classification. (Adapted from: Schacter and Tulving, 1994; Willingham, 1997; Budson and Price, 2001; Ashby and O’Brien, 2005)

\section{Memory Systems}

A 'memory system' is "a way in which the brain process information that is available for use at a later time, with or without conscious awareness" (Budson and Price, 2001). Of the various memory systems theorized, they can be categorized based on their length of storage and our conscious awareness of the memories in the system, and can be dissociated from the other systems (Schacter and Tulving, 1994). The length of storage can be either longterm or short-term memory, while our awareness is either explicit or implicit. Systems associated with conscious awareness are considered explicit, while others that are associated with behaviour changes or unconscious action are designated as implicit.

In Schacter and Tulving's (1994) memory system classification system, the only short-term memory system mentioned is working memory. However, the actual duration (and even existence) of short-term memory is debated by memory researchers. The conventional assumption is that short-term memory is limited to a few seconds and/or seven plus or minus two memory items (Banich, 2004, p. 239). Working memory is considered to be an explicit, declarative system as it requires "active conscious participation" (Ashby and O'Brien, 2005). Budson and Price (2001) give an example of "silently repeating a seven-digit phone number until you have a chance to write it down." Banich (2004, p. 239) affirms that the most common working memory task would be a digit-span task where items have to be reversed (i.e., repeating a sequence of numbers in reverse order). Ashby and O'Brien (2005) suggest that working memory is crucial for rule-based categorical learning, as assessed in the Wisconsin Card Sorting Test. Functional imaging studies suggest that working memory performance relies on the prefrontal cortex and basal ganglia (Ashby and O'Brien, 2005). Budson and Price (2005) list disorders such as Alzheimer's, Parkinson's, and Huntington's disease, as well as schizophrenia as having been shown to impair working memory, in addition to traumatic brain injuries. Attentional disorders including attention-deficit hyperactivity disorder, obsessive-compulsive disorder, schizophrenia, and depression can also impair working memory (Budson and Price, 2005).
When discussing long-term memory disorders, there are five memory systems theorized (Budson and Price, 2001). These systems are either implicit in nature, as in classical conditioning, priming, and procedural memory, or explicit, as in episodic and semantic memory.

Classical conditioning is the theory behind the traditional stimulus-response relationship, best observed through Pavlov's experiments (Budson and Price, 2001). Classical condition relies primarily on the amygdala and the cerebellum, for emotional and skeletal motor responses respectively (Willingham, 1997). Classically, amnesic patients are not impaired in conditioning tasks, though there are some exceptions. Budson and Price (2001) reveal that damage to this system can occur in progressive stages of Alzheimer's disease and in some Korsakoff syndrome (from mediodorsal thalamic nuclear damage) patients, as well as in cerebellar degenerative diseases. Conditioning a tone to be associated with a puff of air to the eye, causing the subject to blink, is a common test of classical conditioning (Shimamura, 2003). Amnesic subjects in this task often retain the eye blink response for up to 24 hours, though they did not recognize the testing apparatus.

Priming is another type of long-term, implicit, memory and is similar to the procedural and semantic memory systems (Tulving and Schacter, 1990). According to Willingham (1997), the basis for this system is that subjects are asked to study materials, then after a period of time, are given a task that utilizes the same study materials. Tulving and Schacter (1990) report that priming appears to be unaffected in most amnesic patients, neural degeneration associated with aging, and drug effects. Anatomically, priming is associated with the neocortical regions that mediate the priming sensory information, i.e. the occipital lobe for visual priming, though large lesions to the sensory association cortex can also cause deficits in priming (Budson and Price, 2001). Due to the implicit and covert nature of priming, impairments in this system are unlikely to be noticed outside of an experimental setting.

Last of the implicit memory systems is procedural memory, which "underlies changes in skillful performance and appropriate responding to stimuli" (Tulving and Schacter, 1990). A prime example of a procedural memory 


\section{R. Madan / Eureka 2 (2011)}

task is learning to ride a bike. Budson and Price (2005) report that functional neuroimaging has found procedural memory to be rooted in the basal ganglia, cerebellum, and the supplementary motor area. As a result, diseases that affect the basal ganglia, including Parkinson's disease and Huntington's disease, can impair this memory system (Budson and Price, 2001). Exclusive cerebellar damage, as found in some strokes and tumours, can also cause deficits to this memory system.

Many examples of procedural memory-based tasks have been used in research of the dissociations between memory systems. In these tasks amnesic patients are able to learn motor skills or be successfully primed without being able to recall having learned the tasks at all, though the use of explicit memory does greatly enhance an individual's ability to acquire procedural memory-based skills (Willingham, Nissen, and Bullemer, 1989). Corkin (2002) illustrates an example of this learning in experimental practices in H.M.'s improving performance over time in a mirror-tracing task (tracing the outline of a given shape, usually a 6-pointed star, within a narrow boundary while looking at the reflection of the page in the mirror). This type of learning has been further extended to involve mirror-reading (reading mirror-reversed text), though the amnesic patients were still impaired in recognizing the words (Willingham et al., 1989). In current neuropsychological assessments, the aforementioned tasks have been succeeded by the serial reaction time task (Ashby and O'Brien, 2005), involving the subject pressing keys as quickly as possible as a response to stimuli presented on a screen. In this task, the response time diminishes significantly when sequences are introduced, even when the subject is unaware that there is a sequence.

Despite the dissociation between procedural memory and the other memory systems, it can be more difficult to isolate procedural memory than is commonly stated. One example of this is demonstrated in Xu and Corkin's (2001) attempts to teach the H.M. to solve the Tower of Hanoi puzzle. In this study, even though H.M. did show some improvement throughout the day in the task under certain conditions, the learning was not carried over to the next day. Another example of this problem has been shown in studies with amnesics playing the computer game Tetris and then later having hypnagogic images of the game despite having no conscious recollection of having played the game at all. However, learning is improvished when compared with novice controls, suggesting a reliance of Tetris proficiency on declarative memory in addition to the procedural memory system (Stickgold, Malia, Maguire, Roddenberry, and O'Conner, 2000).

The remaining two memory systems are classified as explicit in nature, and are collectively referred to as declarative memory, although there is some debate between memory disorder researchers as to classification of these memory systems. Shimamura (2003) describes "memories that are tied to one's autobiography" as episodic memory, while semantic memory as "the vast database of factual knowledge stored in memory". These two memory systems also appear to be lateralized in the brain, as language processes are primarily located in the left cerebral hemisphere, while spatial processing is in the right hemisphere. Shimamura (2003) suggests that since episodic memory is largely spatial in nature, damage to the right hemisphere causes more deficits in this memory system than in the semantic memory system. In concordance, semantic memory impairments are often associated with lesions to the left cerebral hemisphere.

Deficits in episodic memory are the most common aspect of the amnesic syndrome. Squire and Zola (1998) describe episodic memory as "the capacity for recollecting happenings from the past, for remembering events that occurred in particular spatial and temporal contexts." Anatomically, episodic memory is based around the Papez circuit, originally proposed by Papez in 1937 (Budson and Price, 2001). This neuroanatomical circuit illustrates how information is transferred in and out of the hippocampus via the cingulate gyrus, in the medial temporal lobes. Squire et al. (2001) also report that "the hippocampus itself is important for memory for a relatively short period of time after learning and that the adjacent perirhinal and parahippocampal cortices remain important for a longer time." Some of the disorders that disrupt this memory system include: Alzheimer's disease, Korsakoff's syndrome, Herpes encephalitis, severe hypoxia, vascular disorders, transient global and epileptic amnesias, and head injuries (Kopelman, 2002). These syndromes are the primary causes of anterograde amnesia as well as some retrograde amnesias.

Budson and Price (2001) also notes that some aetiologies such as vascular and frontal lobe dementia are also known to cause distortions of episodic memory, as opposed to outright memory failure. In these cases, the origin of the deficit is not caused by damage to the Papez circuit, but rather damage in the frontal lobes. In the analogy proposed by Budson and Price (2001), the frontal lobes act as a 'file clerk', with the medial temporal lobes acting as the 'recent memory file cabinet' and other cortical regions serving as a 'remote memory file cabinet'. If the frontal lobe has been damaged, improper filing occurs and the memories may be distorted and inaccurate. Some forms of amnesia, caused by solely by frontal lobe pathology, are thus characterised mainly by faulty retrieval processes (i.e. head injury with exclusive frontal lobe damage) and memory distortions (Kopelman, 2002). In other diseases, such as Alzheimer's disease, both damage to the temporal lobe and frontal lobe regions co-occur with progression of the disease, causing genuine memory loss along with memory distortions.

Depending on the exact nature of the episodic memory deficits, it is possible to develop strategies to overcome the impairments. Huppert and Piercy (1978) report that patients with Korsakoff's syndrome were able to achieve picture recognition performance comparable to the control subjects after observing the stimulus for 4-8 times longer than the controls. They suggest the extra time allowed the Korsakoff patients to achieve the same level of learning that the controls reached in less time. The deficits found on the patients only affect the initial learning and not the subsequent recall (Huppert and Piercy, 1978). However, Squire and Zola (1998) suggest that the mechanism for this process may rely on the semantic memory system. 


\section{R. Madan / Eureka 2 (2011)}

The last memory system, semantic memory, is based in understanding concepts and general knowlege. Studies of semantic memory have localized it to the inferolateral temporal lobe and the memory system is commonly impaired in Alzheimer's disease and semantic dementia patients (Budson and Price, 2005). In the progression of Alzheimer's disease, the most vulnerable and first affected system appears to be episodic memory and then, in later stages, also semantic memory. (Kensinger, Ullman, and Corkin, 2001). Tulving and Markowitsch (1998) believe this is because the episodic memory system is a special case of semantic memory. Still, not all memory researchers agree with this view (Squire and Zola, 1998).

Semantic memory impairments can be seen in the inability to name items that were previously well-known, as well as impoverished general knowledge (Budson and Price, 2001). In cases with mild semantic memory deficits, difficulties in naming items of a certain category may occur, as seen in propagnosia, where the patient's ability to recognize faces is impaired (Shimamura, 2003). In propagnosia, brain damage is limited to the fusiform gyrus, which is specialized for facial recognition. These patients can still recognize familiar individuals by their voice. Other mild impairments may involve inabilities in naming animals or tools (Budson and Price, 2001). As outlined by Kopelman (2002) there is still, there is some dispute as to the actual mechanisms behind some of the dissociations found between different categories.

Semantic memory is also believed to be partially dependent on the medial temporal lobe, commonly damaged in amnesics experiencing episodic memory deficits (Squire and Zola, 1998). This view is supported by studies showing that episodic and semantic memory alike are impaired in patients with hippocampal damage as both memory systems share many commonalities (Tulving and Markowitsch, 1998). However, Squire and Zola (1998) state that in patients with frontal lobe damage, deficits in episodic memory are exhibited while semantic memory appears to be intact. Even still, semantic memory does appear to be partially dissociable from the hippocampus, as seen in studies of H.M. (Kensinger et al., 2001). In studies of H.M. it appears that episodic memory aids in the initial learning of semantic information, but is no longer needed for the retrieval, relying on neocortical regions rather than the hippocampus.

Similarly to episodic memory, even with semantic impairments, it is possible to gain and acquire new information over prolonged periods of time. H.M. was able to draw an accurate floor plan of his home that he moved to after the onset of his amnesia (Corkin, 2002). O'Kane et al. (2004) also report that H.M. was able to learn new famous names via slow-learning and extended repetitions. These findings suggest that less effective, alternative mechanisms for semantic memory-based learning can still occur after brain damage has occurred.

In further analyzing the declarative memory systems, through recognition tasks a particularly intriguing instance has arisen -- familiarity. These tasks are based on the 'remember-know procedure' designed to differentiate between recollecting (through retrieval of episodic details) the word presented and feelings of familiarity of the word (Quamme, Yonelinas, and Norman, 2007). While tests of recollection-based recognition require the hippocampus, familiarity judgements appear to be spared in cases of hippocampal amnesia (Spiers et al., 2001). Stark and Squire (2000) ruled out priming as a mechanism for this effect in their study. Quamme et al. (2007) instead propose that limited medial-temporal lobe amnesia can be associated with spared capacity for familiarity discriminations. Quamme et al.'s (2007) study also was able to mimic the effect found in hippocampal amnesics with undergraduate students instructed to use a specific familiarity-based strategy. Research into this mechanism is still just beginning and will be an key step in memory research differentiating the two declarative memory systems.

Though several of these memory systems are not commonly associated with the amnesic syndrome, such as classical conditioning deficits, their resulting symptoms can be classified as amnesic in nature (Budson and Price, 2001). As stated by Willingham (1997), other researchers have proposed further breakdowns to the previously described memory systems. The deficits presented by the various memory system impediments found in diseases and syndromes associated with organic amnesia can be quite diverse and it is unlikely for an individual to experience impairments in multiple memory systems.

\section{Conclusion}

McConnell (1989, p. 278) describes the effect of amnesia as "the process by which [information] is physically erased from your memory banks, blocked off from easy access, or prevented from being stored in the first place." Even more importantly, as described by Corkin (2002), "amnesia is not an all-or-nothing condition," though Budson and Price (2001) mention that some diseases such as Alzheimer's can impair multiple memory systems. In real-life, most situations rely on multiple memory systems (Ashby and O'Brien, 2005).

Organic amnesias are memory disturbances that are caused by specific malfunctions in the brain (DANA Foundation, 2007). Shimamura (2003) mentions that the location of the brain lesion is not the most important feature, but rather the size of the lesion, that determines the severity of the impairment. Memories are stored throughout the brain, and as a result damage to any localized brain region only causes limited memory deficits. However, a large brain lesion would lead to greater and more profound memory impairments. The precise deficits found in individuals with amnesia can vary a great deal, although there are some commonalties, such as the "severe loss of post- and pre-morbid episodic memories in virtually all patients with bilateral hippocampal damage" (Spiers et al., 2001). As mentioned by Spiers et al. (2001), other features may vary, such as the semantic memory and familiarity-based recognition.

Though there is a great diversity in disorders involving organic amnesia symptoms and there are several memory systems that can be affected, often the resulting impairments can be diminished through the use of compensatory strategies. 


\section{R. Madan / Eureka 2 (2011)}

\section{References}

Ashby, F.G and O'Brien, J.B. (2005). Category learning and multiple memory systems. TRENDS in Cognitive Sciences, 9(2), 83-89.

Banich, M.T. (2004). Cognitive Neuroscience and Neuropsychology (2nd ed.). Boston, MA: Houghton Mifflin.

Baxendale, S. (2004). Memories aren't made of this: amnesia the movies. British Medical Journal, 329, 1480-1483.

Budson, A.E. and Price, B.H. (2001) Memory: Clinical Disorders. Encyclopedia of Life Sciences. Chichester: John Wiley and Sons, Ltd.

Budson, A.E. and Price, B.H. (2005). Memory Dysfunction. New England Journal of Medicine, 352, 692-699.

Corkin, S. (2002). What's new with the amnesic patient H.M.? Nature Reviews Neuroscience, 3, 153-160.

DANA Foundation. (2007). Amnesias. The DANA Guide to Brain Health. Retrieved October 17, 2007, from http://www.dana.org/brainhealth/ detail. aspx?id=4716.

Fujiwara, E., Brand, M., Kracht, L., Kessler, J., Diebel, A., Netz, J., Markowitsch, H.J. (2008). Functional retrograde amnesia: a multiple case study. Cortex, 44, 29-45.

Gold, P.E. (2006). The many faces of amnesia. Learning and Memory, 13, 506-514.

Huppert, F.A and Piercy, M. (1978). Dissociation between learning and remembering in organic amnesia. Nature, 275, 317-318.

Kapur, N. (1999). Syndromes of retrograde amnesia: A conceptual and empirical synthesis. Psychological Bulletin, 125(6), 800-825.

Kensinger, E.A., Ullman, M.T., and Corkin, S. (2001). Bilateral medial temporal lobe damage does not affect lexical or grammatical processing: Evidence from amnesic Patient H.M. Hippocampus, 11, 347-360.

Kopelman, M.D. (2002). Disorders of memory. Brain, 125, 2152-2190.

McConnell, J.V. (1989). Understanding Human Behavior (6th ed.). New York: Holt, Rinehart and Winston.

O'Kane, G., Kensinger, E.A., Corkin, S. (2004). Evidence for semantic learning in profound amnesia: An investigation with Patient H.M. Hippocampus, 14, 417-425.

Passer, M.W., and Smith, R.E. (2007). Psychology: The Science of Mind and Behavior (3rd Ed.). New York: McGraw-Hill.
Quamme, J.R., Yonelinas, A.P., Norman, K.A. (2007). Effect of unitization on associative recognition in amnesia. Hippocampus, 17, 192-200.

Schacter, D.L. and Tulving, E. (1994). Memory Systems. Cambridge, MA: MIT Press.

Scoville, W.M. and Milner, B. (1957). Loss of recent memory after bilateral hippocampal lesions. Journal of Neurology, Neurosurgery and Psychiatry, 20, 11-21.

Shimamura, A.P. (1992). Organic Amnesia, from L.R. Squire (Ed.), Encyclopedia of Learning and Memory, pp. 30-35. New York: Macmillan.

Shimamura, A.P. (2003). Neural basis of memory: Systems level. In L. Nadel (Ed.), Encyclopedia of Cognitive Science, London: Macmillan.

Simons, J.S. and Graham, K.S. (2000). Amnesia, from G. Fink (Ed.), Encyclopedia of Stress, pp. 176-178. San Diego: Academic Press.

Stark, C.E. and Squire, L.R. (2000). Recognition memory and familiarity judgements in severe amnesia: No evidence for a contribution of repetition priming. Behavioural Neuroscience, 114(3), 459-467.

Stickgold, R., Malia, R., Maguire, D., Roddenberry, D., and O'Conner, M. (2002). Replaying the game: Hypnagogic images in normals and amnesics. Science, 290, 350-353.

Spiers, H.J., Maguire, E.A., and Burgess, N. (2001). Hippocampal Amnesia. Neurocase, 7, 357-382.

Squire, L.R., Clark, R.E., and Knowlton, B.J. (2001) Retrograde Amnesia. Hippocampus, 11, 50-55.

Squire, L.R. and Zola, S.M. (1998). Episodic Memory, Semantic Memory, and Amnesia. Hippocampus, 8, 205-211.

Tulving, E. and Markowitsch, H.J. (1998). Episodic and declarative memory: Role of the hippocampus. Hippocampus, 8, 198-204.

Tulving, E. and Schacter, D.L. (1990). Priming and human memory systems. Science, 247, 301-306.

Willingham, D.B., Nissen, M.J., and Bullemer, P. (1989). On the development of procedural knowledge. Journal of Experimental Psychology: Learning, Memory, and Cognition, 15(6), 1047-1060.

Willingham, D.B. (1997). Systems of memory in the human brain. Neuron, 18, 5-8.

Xu, Y. and Corkin, S. (2001). H.M. revisits the Tower of Hanoi puzzle. Neuropsychology, 15(1), 69-79. 